\title{
Improving Iterative Solutions of the Electric-Field Integral Equation Via Transformations Into Normal Equations
}

\author{
Ö Ergül \& L. Gürel
}

To cite this article: Ö Ergül \& L. Gürel (2010) Improving Iterative Solutions of the Electric-Field Integral Equation Via Transformations Into Normal Equations, Journal of Electromagnetic Waves and Applications, 24:16, 2129-2138, DOI: 10.1163/156939310793699082

To link to this article: http://dx.doi.org/10.1163/156939310793699082

曲 Published online: 03 Apr 2012.

Submit your article to this journal $\sqsubset$

Џ Article views: 30

Q View related articles ¿

Citing articles: 7 View citing articles ๔ 


\title{
IMPROVING ITERATIVE SOLUTIONS OF THE ELECTRIC- FIELD INTEGRAL EQUATION VIA TRANSFORMA- TIONS INTO NORMAL EQUATIONS
}

\author{
Ö. Ergül \\ Department of Mathematics and Statistics \\ University of Strathclyde, Glasgow, UK

\section{Gürel ${ }^{\dagger}$} \\ Department of Electrical and Electronics Engineering \\ Bilkent University, Bilkent, Ankara, Turkey
}

\begin{abstract}
We consider the solution of electromagnetics problems involving perfectly conducting objects formulated with the electric-field integral equation (EFIE). Dense matrix equations obtained from the discretization of EFIE are solved iteratively by the generalized minimal residual (GMRES) algorithm accelerated with a parallel multilevel fast multipole algorithm. We show that the number of iterations is halved by transforming the original matrix equations into normal equations. This way, memory required for the GMRES algorithm is reduced by more than $50 \%$, which is significant when the problem size is large.
\end{abstract}

\section{INTRODUCTION}

Surface integral equations, namely, the electric-field integral equation (EFIE), the magnetic-field integral equation (MFIE), and the combined-field integral equation (CFIE), are commonly used to formulate electromagnetics problems involving perfectly conducting objects $[1,10]$. Discretizations of those formulations lead to dense matrix equations, which can be solved iteratively by using Krylovsubspace algorithms [14]. MFIE and CFIE are applicable only to closed surfaces, whereas EFIE can be applied to both open and closed

\footnotetext{
Received 18 July 2010, Accepted 12 October 2010, Scheduled 20 October 2010

Corresponding author: Özgür Ergül (ozgur.ergul@strath.ac.uk).

† Also with Computational Electromagnetics Research Center (BiLCEM), Bilkent University, Bilkent, Ankara, Turkey.
} 
surfaces. Therefore, thin conductors that are modeled as open surfaces must be formulated with EFIE [15,16]. On the other hand, EFIE usually produces ill-conditioned matrix equations that are difficult to solve iteratively $[2,3,21]$, even with acceleration methods such as the multilevel fast multipole algorithm (MLFMA) [4,11, 12, 17-20].

As the problem size grows and the number of unknowns increases, it becomes critical to reduce the iteration counts as much as possible to obtain efficient solutions. Naturally, the number of iterations depends on the type of the iterative solver. In the literature, various Krylov-subspace algorithms, such as conjugate gradient squared (CGS), biconjugate gradient (BiCG), biconjugate gradient stabilized (BiCGStab), generalized minimal residual (GMRES), least-squares QR (LSQR), quasi-minimal residual (QMR), and transpose-free QMR are available to solve non-Hermitian matrix equations derived from the discretization of surface formulations [5-7]. Recently, we showed that LSQR performs better than the other algorithms (except for GMRES) for the solution of scattering problems formulated with EFIE [8]. For a class of problems, only the GMRES algorithm provides faster solutions than LSQR at the cost of increased memory usage. Solutions with LSQR correspond to transforming the original equations into normal equations and solving the resulting Hermitian systems with a conjugate gradient (CG) algorithm [5]. We also showed that the improved convergence of LSQR is due to the favourable properties of the transformation into normal equations for EFIE [8].

For iterative solutions of EFIE, GMRES is usually an optimal algorithm in terms of the solution time. In addition to the improved convergence rate, GMRES requires only one matrixvector multiplication per iteration, as opposed to the other iterative algorithms. However, as a major disadvantage, GMRES requires larger memory than the others. Memory used by GMRES increases linearly as the iterations proceed, and it becomes substantial when the problem size is large. For large problems, however, memory is usually more critical than the processing time and the available memory should be used economically. It is possible to set a maximum memory for GMRES and restart the algorithm whenever required, i.e., when there is no more available memory. On the other hand, restarting GMRES reduces the fast convergence rate offered by this algorithm.

In this paper, we employ GMRES without a restart for the solution of normal equations obtained with EFIE. Transforming an original matrix equation into a normal equation, the iterative convergence of GMRES is improved and the number of iterations is approximately halved. Since the number of matrix-vector multiplications per iteration is doubled at the same time, the processing time does not decrease 
significantly. On the other hand, for large problems, memory required for GMRES is reduced by more than 50\%, which is significant compared to the total memory used for the solution.

\section{TRANSFORMATION INTO NORMAL EQUATIONS}

Discretization of EFIE leads to $N \times N$ dense matrix equations in the form of

$$
\overline{\mathbf{Z}} \cdot \mathbf{a}=\mathbf{v},
$$

where $\overline{\mathbf{Z}}$ represents the impedance matrix, $\mathbf{v}$ represents the right-handside vector due to excitations, and $\mathbf{a}$ is the unknown coefficient vector. In frequency domain, $\overline{\mathbf{Z}}$, $\mathbf{a}$, and $\mathbf{v}$ involve complex numbers. Using a Galerkin scheme for the discretization of EFIE, $\overline{\mathbf{Z}}$ is symmetric, but not Hermitian. Instead of solving the original matrix equation in (1), we can solve the normal equation

$$
\overline{\mathbf{Z}}^{*} \cdot \overline{\mathbf{Z}} \cdot \mathbf{a}=\overline{\mathbf{Z}}^{*} \cdot \mathbf{v}
$$

where ' $*$ ' represents the complex-conjugate operation. We note that the transformed matrix equation involves a Hermitian matrix, i.e., $\overline{\mathbf{Y}}=\overline{\mathbf{Z}}^{*} \cdot \overline{\mathbf{Z}}$ is symmetric and $\overline{\mathbf{Y}}^{*}=\overline{\mathbf{Y}}$.

For large-scale problems, we accelerate the iterative solutions using MLFMA [4]. For an $N \times N$ dense matrix equation, MLFMA performs the matrix-vector multiplications required by the iterative solver in $\mathcal{O}(N \log N)$ time using $\mathcal{O}(N \log N)$ memory. For the normal equation in (2), matrix-vector multiplications are performed as

$$
\mathbf{y}^{*}=\overline{\mathbf{Z}} \cdot(\overline{\mathbf{Z}} \cdot \mathbf{x})^{*}
$$

where $\mathbf{x}$ and $\mathbf{y}$ are input and output vectors, respectively. This way, an ordinary MLFMA constructed for the original matrix $\overline{\mathbf{Z}}$ is used twice without any modification.

It is well-known that the transformation into normal equations increases the condition number of the linear system. Specifically, the condition number of the transformed matrix $\overline{\mathbf{Z}}^{*} \cdot \overline{\mathbf{Z}}$ is the square of the condition number of the original matrix $\overline{\mathbf{Z}}$. On the other hand, the convergence rate of an iterative solution is not strictly correlated with the condition number. In fact, as discussed in [8], transformations into normal equations can accelerate the iterative solutions of EFIE. As an example, we present the solution of a scattering problem involving a $5 \lambda \times 2.5 \lambda \times 0.5 \lambda$ perfectly conducting box in free space, where $\lambda$ is the wavelength. As depicted in Fig. 1(a), the box is illuminated by a plane wave propagating in the $-z$ direction with the electric field polarized in the $y$ direction. Since the object is modelled as a closed surface, the scattering problem can be formulated with both EFIE 


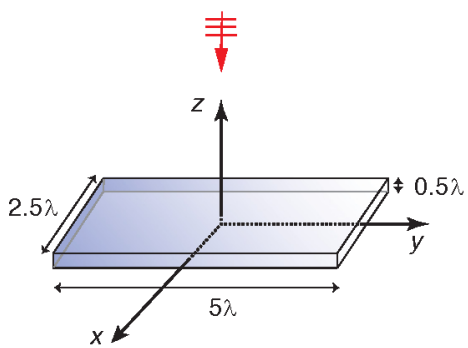

(a)

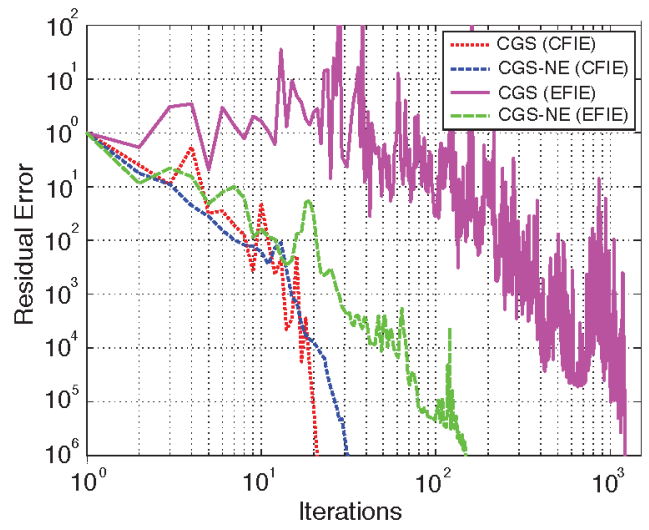

(b)

Figure 1. (a) Scattering problem involving a $5 \lambda \times 2.5 \lambda \times 0.5 \lambda$ perfectly conducting box illuminated by a plane wave propagating in the $-z$ direction with the electric field polarized in the $y$ direction. Iterative solutions of the scattering problem in Fig. 1(a), when the problem is formulated with EFIE and CFIE, and CGS is applied to the ordinary and normal equations.

and CFIE. Discretizations of the integral equations and the box with the Rao-Wilton-Glisson (RWG) functions on $\lambda / 10$ triangles lead to matrix equations involving 11,256 unknowns. Fig. 1(b) depicts the iterative solutions of the scattering problem when the CGS algorithm is applied to the ordinary and transformed (normal) matrix equations. In each case, the residual error is plotted as a function of iterations until the error is decreased to less than $10^{-6}$. Fig. 1(b) shows that, with the transformation into normal equations, CFIE has a slower convergence compared to its ordinary solution. Specifically, for the CFIE formulation, the number of iterations to reduce the residual error to less than $10^{-6}$ is 21 and 32 when CGS is applied to the ordinary and normal equations, respectively. However, in the case of EFIE, the transformation leads to faster convergence than the ordinary solution, and the number of iterations is drastically reduced from 1211 to 149 . In other words, the solution of EFIE is significantly improved by the transformation into normal equations.

As presented in Fig. 1, favourable properties of the transformation into normal equations for EFIE are clearly observed when the solutions are performed by CGS. On the other hand, in terms of the processing time, GMRES is usually the optimal iterative solver for EFIE [13]. Hence, it is essential to demonstrate the improvement 


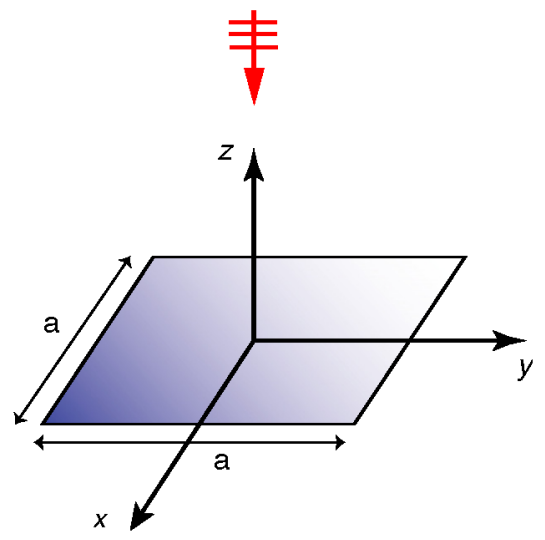

Figure 2. Scattering problems involving an $a \times a$ square patch.

by the transformation when the solutions are performed by GMRES. In fact, as demonstrated in the next section, the transformation into normal equations can substantially reduce the memory required by GMRES.

\section{RESULTS}

In order to demonstrate the improved GMRES solutions of EFIE via transformations into normal equations, we consider the solution of scattering problems involving a square perfectly conducting patch, as depicted in Fig. 2. The size of the patch $a$ changes from $12 \lambda$ to $30 \lambda$ and it is located in free space. The patch is illuminated by a plane wave with normal incidence. Discretizations of EFIE and the patch geometry with the RWG functions on $\lambda / 10$ triangles lead to matrix equations involving 49,200 to 310,382 unknowns. Problems are solved by using a parallel implementation of MLFMA [9] on a cluster of 16 AMD Opteron 870 processors. Table 1 lists the number of iterations for $10^{-6}$ residual error, solution time in seconds (s), and memory in megabytes (MB) per processor, when GMRES is used to solve the original and transformed (normal) matrix equations. Only the memory required for GMRES is considered. Applying GMRES on normal equations (denoted as GMRES-NE), the number of iterations is reduced by $53-59 \%$, compared to the conventional solutions. The processing time, however, does not decrease by the same amount, since the number of matrix-vector multiplications per iteration is doubled for GMRES-NE. Nevertheless, for the largest problem discretized with 310,383 unknowns, the processing time is reduced by $17 \%$, while the 
Table 1. Solutions of scattering problems involving a patch geometry of various sizes.

\begin{tabular}{|c|c|c|c|c|}
\hline & & \multicolumn{3}{|c|}{ GMRES } \\
\hline Size & $N$ & Iterations & Time (s) & Memory (MB) \\
\hline \hline $12 \lambda \times 12 \lambda$ & 49,200 & 620 & 568 & 30 \\
\hline $18 \lambda \times 18 \lambda$ & 111,405 & 776 & 2372 & 81 \\
\hline $20 \lambda \times 20 \lambda$ & 137,792 & 793 & 2635 & 107 \\
\hline $25 \lambda \times 25 \lambda$ & 215,338 & 877 & 4232 & 180 \\
\hline $30 \lambda \times 30 \lambda$ & 310,383 & 991 & 6070 & 290 \\
\hline
\end{tabular}

\begin{tabular}{|c|c|c|c|c|}
\hline & & \multicolumn{3}{|c|}{ GMRES-NE } \\
\hline Size & $N$ & Iterations & Time (s) & Memory (MB) \\
\hline \hline $12 \lambda \times 12 \lambda$ & 49,200 & 254 & 445 & 13 \\
\hline $18 \lambda \times 18 \lambda$ & 111,405 & 335 & 1959 & 36 \\
\hline $20 \lambda \times 20 \lambda$ & 137,792 & 360 & 2269 & 49 \\
\hline $25 \lambda \times 25 \lambda$ & 215,338 & 413 & 3620 & 87 \\
\hline $30 \lambda \times 30 \lambda$ & 310,383 & 467 & 5016 & 139 \\
\hline
\end{tabular}

number of matrix-vector multiplications decreases only by $6 \%$. This is because the cost of GMRES itself increases quadratically with the number of iterations [6]. Hence reducing the number of iterations is preferable, even when the number of matrix-vector multiplications does not decrease significantly. Finally, Table 1 shows that memory required for GMRES is reduced by $52-57 \%$. For the largest problem, MLFMA itself requires only $135 \mathrm{MB}$ memory per processor. Then, the total memory usage (including GMRES and MLFMA) per processor is reduced from $425 \mathrm{MB}$ to $274 \mathrm{MB}$, just by solving the normal equation instead of the original equation with GMRES.

The transformation into normal equations proposed in this paper reduces the memory required by GMRES without deteriorating its fast convergence rate. In fact, as demonstrated in the previous example, the processing time is slightly reduced by the transformation. A common practice in the literature to reduce memory required for GMRES is restarting the algorithm, which may negatively affect the convergence rate. As an example, the scattering problem involving a $20 \lambda \times 20 \lambda$ patch discretized with 137,792 unknowns is solved by a restarted GMRES. The restart parameter is adjusted carefully such 


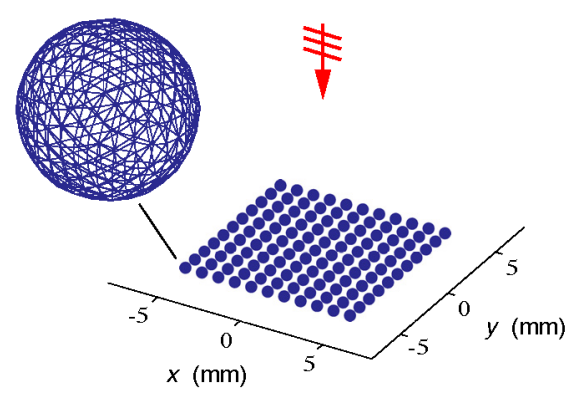

(a)

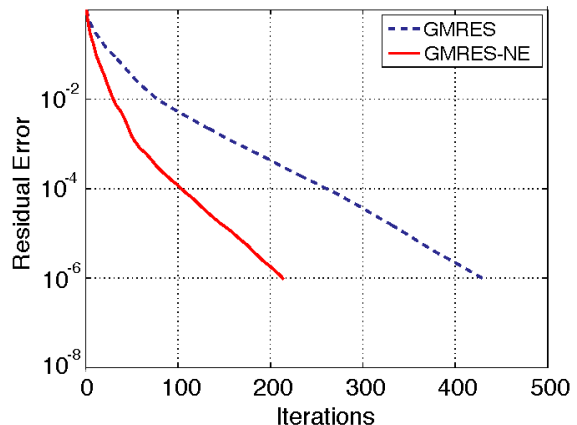

(b)

Figure 3. (a) Scattering problem involving a wall of $11 \times 11$ perfectly conducting spheres illuminated by a plane wave. (b) Convergence histories when the scattering problem is solved with GMRES applied to the original and transformed matrix equations.

that the maximum memory used by GMRES is limited to $49 \mathrm{MB}$, i.e., the amount of memory used by GMRES applied to the normal equations. Using the restarted GMRES, the number of iterations is increased to 900 and the solution time is increased 2877 seconds, which is $27 \%$ larger than the processing time of GMRES-NE. Nevertheless, the transformation into normal equations does not conflict with the restarting ability of GMRES, and the normal formulations can also be solved via a restarted GMRES to further limit the memory usage, and at the same time, to benefit from the favourable properties of the transformation.

Finally, we present the solution of a scattering problem involving a wall of perfectly conducting spheres. As depicted in Fig. 3(a), the wall is a two-dimensional array that consists of $11 \times 11$ spheres and it is illuminated by a plane wave with normal incidence (normal to the plane of the array) at $500 \mathrm{GHz}$. Spheres have a radius of $0.3 \mathrm{~mm}$ and they are arranged regularly with $1 \mathrm{~mm}$ periodicity in both $x$ and $y$ directions. Hence, the overall size of the wall is $5.3 \times 5.3 \times 0.6 \mathrm{~mm}$. The problem is discretized with 112,530 RWG functions defined on $\lambda / 10$ triangles. Fig. 3(b) presents convergence histories when the problem is solved with GMRES applied to the original and transformed matrix equations. In both cases, 6-level sequential MLFMA is employed to accelerate the matrix-vector multiplications. For the original matrix equations, the number of iterations required for less than $10^{-6}$ residual error is 429. Transforming the matrix equation increases the convergence rate and the number of iterations is reduced to 213 . 
Consequently, memory required for GMRES is reduced from $750 \mathrm{MB}$ to $390 \mathrm{MB}$, and the total memory (including GMRES and MLFMA) is reduced from $1447 \mathrm{MB}$ to $1087 \mathrm{MB}$ by solving the normal equation instead of the original equation.

\section{CONCLUSION}

Iterative solutions of EFIE can be obtained in fewer iterations with a simple transformation of the original matrix equations into normal equations. This way, memory required for the GMRES algorithm is reduced significantly, even though the solution time is not decreased by the same amount. The transformation is easy to apply by using the existing implementations for the ordinary matrix-vector multiplications. Finally, the proposed transformation is not an alternative method to preconditioning, and preconditioners that are useful for the ordinary solutions of EFIE can also be employed to improve the transformed solutions.

\section{ACKNOWLEDGMENT}

This work was supported by the Scientific and Technical Research Council of Turkey (TUBITAK) under Research Grant 107E136, by the Turkish Academy of Sciences in the framework of the Young Scientist Award Program (LG/TUBA-GEBIP/2002-1-12), and by contracts from ASELSAN and SSM. Özgür Ergül was also supported by a Research Starter Grant provided by the Faculty of Science at the University of Strathclyde.

\section{REFERENCES}

1. Poggio, A. J. and E. K. Miller, "Integral equation solutions of three-dimensional scattering problems," Computer Techniques for Electromagnetics, R. Mittra (ed.), Chap. 4, Pergamon Press, Oxford, 1973.

2. Wilton, D. R. and J. E. Wheeler III, "Comparison of convergence rates of the conjugate gradient method applied to various integral equation formulations," Progress In Electromagnetics Research, Vol. 5, 131-158, 1991.

3. Ergül, Ö. and L. Gürel, "Iterative solutions of hybrid integral equations for coexisting open and closed surfaces," IEEE Trans. Antennas Propag., Vol. 57, No. 6, 1751-1758, Jun. 2009. 
4. Song, J., C.-C. Lu, and W. C. Chew, "Multilevel fast multipole algorithm for electromagnetic scattering by large complex objects," IEEE Trans. Antennas Propag., Vol. 45, No. 10, 14881493, Oct. 1997.

5. Paige, C. C. and M. A. Saunders, "LSQR: An algorithm for sparse linear equations and sparse least squares," ACM Trans. Math. Software, Vol. 8, No. 1, 43-71, Mar. 1982.

6. Saad, Y. and M. H. Schultz, "GMRES: A generalized minimal residual algorithm for solving nonsymmetric linear systems," SIAM J. Sci. Stat. Comput., Vol. 7, No. 3, 856-869, 1986.

7. Van Der Vorst, H. A., "Bi-CGSTAB: A fast and smoothly converging variant of $\mathrm{Bi}-\mathrm{CG}$ for the solution of nonsymmetric linear systems," SIAM J. Sci. Stat. Comput., Vol. 13, No. 2, 631644, Mar. 1992

8. Ergül, Ö. and L. Gürel, "Efficient solution of the electric-field integral equation using the iterative LSQR algorithm," IEEE Antennas Wireless Propag. Lett., Vol. 7, 36-39, 2008.

9. Ergül, Ö. and L. Gürel, "A hierarchical partitioning strategy for efficient parallelization of the multilevel fast multipole algorithm," IEEE Trans. Antennas Propag., Vol. 57, No. 6, 1740-1750, Jun. 2009.

10. Nie, X.-C., L.-W. Li, and N. Yuan, "Precorrected-Fft algorithm for solving combined field integral equations in electromagnetic scattering," Journal of Electromagnetic Waves and Applications, Vol. 16, No. 8, 1171-1187, 2002.

11. Wang, P. and Y. Xie, "Scattering and radiation problem of surface/surface junction structure with multilevel fast multipole algorithm," Journal of Electromagnetic Waves and Applications, Vol. 20, No. 15, 2189-2200, 2006.

12. Taboada, J. M., M. G. Araujo, J. M. Bertolo, L. Landesa, F. Obelleiro, and J. L. Rodriguez, "MLFMA-FFT parallel algorithm for the solution of large-scale problems in electromagnetics," Progress In Electromagnetics Research, Vol. 105, 15-30, 2010.

13. Ergül, Ö., T. Malas, and L. Gürel, "Solutions of largescale electromagnetics problems using an iterative inner-outer scheme with ordinary and approximate multilevel fast multipole algorithms," Progress In Electromagnetics Research, Vol. 106, 203-223, 2010.

14. Carpentieri, B., "Fast iterative solution methods in electromagnetic scattering," Progress In Electromagnetics Research, Vol. 79, 151-178, 2008. 
15. Gürel, L. and Ö. Ergül, "Design and simulation of circular arrays of trapezoidal-tooth log-periodic antennas via genetic optimization," Progress In Electromagnetics Research, Vol. 85, 243-260, 2008.

16. Chen, Y., S. Yang, S. He, and Z.-P. Nie, "Design and analysis of wideband planar monopole antennas using the multilevel fast multipole algorithm," Progress In Electromagnetics Research B, Vol. 15, 95-112, 2009.

17. Yang, M.-L. and X.-Q. Sheng, "Parallel high-order FE-BIMLFMA for scattering by large and deep coated cavities loaded with obstacles," Journal of Electromagnetic Waves and Applications, Vol. 23, No. 13, 1813-1823, 2009.

18. Ping, X. W., T.-J. Cui, and W. B. Lu, "The combination of bcgstab with multifrontal algorithm to solve febi-mlfma linear systems arising from inhomogeneous electromagnetic scattering problems," Progress In Electromagnetics Research, Vol. 93, 91105, 2009.

19. Peng, Z., X.-Q. Sheng, and F. Yin, "An efficient twofold iterative algorithm of FE-BI-MLFMA using multilevel inverse-based ilu preconditioning," Progress In Electromagnetics Research, Vol. 93, 369-384, 2009.

20. Ouyang, J., F. Yang, S. W. Yang, and Z. P. Nie, "Exact simulation method VSWIE + MLFMA for analysis radiation pattern of probe-feed conformal microstrip antennas and the application of synthesis radiation pattern of conformal array mounted on finite-length PEC circular cylinder with DEs," Journal of Electromagnetic Waves and Applications, Vol. 21, No. 14, 19952008, 2007.

21. Gürel, L., Ö. Ergül, A. Unal, and T. Malas, "Fast and accurate analysis of large metamaterial structures using the multilevel fast multipole algorithm," Progress In Electromagnetics Research, Vol. 95, 179-198, 2009. 